\title{
POLYNOMIALS OF AN INNER FUNCTION WHICH ARE EXPOSED POINTS IN $H^{1}$
}

\author{
JYUNJI INOUE AND TAKAHIKO NAKAZI
}

\begin{abstract}
It is known that if $p(z)$ is an analytic polynomial which has no zeros in the open unit disc and distinct zeros in the unit circle, then $p(z) /\|p(z)\|_{1}$ is an exposed point of the unit ball of the Hardy space $H^{1}$.

In this paper, it is proved that for a bounded analytic function $f$ with $\|f\|_{\infty} \leqslant 1$, $p(f) /\|p(f)\|_{1}$ is also an exposed point.
\end{abstract}

Let $U$ be the open unit disc in the complex plane and let $\partial U$ be the boundary of $U$. If $f$ is analytic in $U$ and $\int_{-\pi}^{\pi} \log \left|f\left(r e^{i \theta}\right)\right| d \theta$ is bounded for $0 \leqslant r<1$, then $f\left(e^{i \theta}\right)$, which we define to be $\lim _{r \rightarrow 1} f\left(r e^{i \theta}\right)$, exists almost everywhere on $\partial U$. If

$$
\lim _{r \rightarrow 1} \int_{-\pi}^{\pi} \log ^{+}\left|f\left(r e^{i \theta}\right)\right| d \theta=\int_{-\pi}^{\pi} \log ^{+}\left|f\left(e^{i \theta}\right)\right| d \theta
$$

then $f$ is said to be in the class $N_{+}$. The set of all boundary functions in $N_{+}$is denoted by $N_{+}$again. For $0<p \leqslant \infty$, the Hardy space $H^{p}$ is defined by $N_{+} \cap L^{p}$. $A$ denotes the disc algebra, that is $A=\{f: f$ is continuous on $\bar{U}$ and analytic in $U\}$. If $h$ in $N_{+}$has the form

$$
h(z)=\exp \left\{\int_{-\pi}^{\pi} \frac{e^{i t}+z}{e^{i t}-z} \frac{\log \left|h\left(e^{i t}\right)\right|}{2 \pi} d t+i \alpha\right\}
$$

for some real $\alpha, h$ is called an outer function. We call $q$ in $N_{+}$an inner function if $\left|q\left(e^{i \theta}\right)\right|=1$ a.e. on $\partial U$.

Let $g$ be a nonzero function in $H^{p}$. Then the following property $(*)$ characterizes that $g$ is an outer function.

(*) Whenever $k g$ belongs to $H^{p}$ for $k$ in $L^{\infty}$ with $k\left(e^{i \theta}\right) \geqslant 0$ a.e. on $U$, then $k$ is a constant function (see [6]).

We can consider a stronger property of $g$ :

(**) Whenever $k g$ belongs to $H^{P}$ for some Lebesgue measurable $k$ with $k\left(e^{i \theta}\right) \geqslant 0$ a.e. on $\partial U$, then $k$ is a constant function.

In [6], the function $g$ with property $(* *)$ is called a $p$-strong outer function. We should remark that deLeeuw and Rudin [1] used the phrase "strong outer function" in a little different context. The $p$-strong outer functions appear to be important in many problems, for example, extremal problems, interpolation problems, Toeplitz

Received by the editors April 22, 1986.

1980 Mathematics Subject Classification (1985 Revision). Primary 30D55, 30D50, 46J15.

Key words and phrases. Exposed points, Hardy spaces, polynomials, inner functions.

This research was partially supported by Grant-in-Aid for Scientific Research, Ministry of Education. 
operators, and prediction theory. In particular, when $\|g\|_{1}=1, g$ is a 1-strong outer function if and only if $g$ is exposed points of the unit ball of $H^{1}$ (see [6]).

Suppose $p(z)=\prod_{j=1}^{n}\left(z+a_{j}\right)$. If $p(z) /\|p(z)\|_{1}$ is an exposed point, then $\left|a_{j}\right| \geqslant 1$ $(j=1, \ldots, n)$ and $a_{i} \neq a_{j}(i \neq j)$ (cf. [1]). It is known that the converse is valid [7], which is also derived from [4 and 5] as follows. If $n=1$, the result follows from Proposition 5 of [4]. Suppose $n>1$ and $\prod_{i=1}^{n-1}\left(z+a_{j}\right) /\left\|\Pi_{j=1}^{n-1}\left(z+a_{j}\right)\right\|_{1}$ is exposed but $p(z) /\|p(z)\|_{1}$ is not. Here we may assume without loss of generality that $\left|a_{j}\right|=1$ for some $j$, say $j=n$. By Proposition 1 of [4], we have an element $k$ in $S_{|p| / p}^{0}$ which can be represented by $\left(e^{i \theta}+a_{n}\right)\left(1+\bar{a}_{n} e^{i \theta}\right) h\left(e^{i \theta}\right)$ for some nonconstant $h$ in $H^{1}$ by Lemma 3 of [5]. Thus we have

$$
k / p=\left(1+\bar{a}_{n} z\right) h / \prod_{j=1}^{n-1}\left(z+a_{j}\right) \geqslant 0 \text { a.e. on } \partial U,
$$

which contradicts the assumption that $\prod_{j=1}^{n-1}\left(z+a_{j}\right) /\left\|\Pi_{j=1}^{n-1}\left(z+a_{j}\right)\right\|_{1}$ is exposed.

Now we wish to prove that $p(f) /\|p(f)\|_{1}$ is an exposed point for the above $p(z)$ and any nonconstant $f$ in $H^{\infty}$ with $\|f\|_{\infty} \leqslant 1$. For $n=1$, this is known [4, Proposition 5]. But we need a new idea to prove it in general.

LEMmA. If $P(z)=\prod_{j=1}^{n}\left(z+a_{j}\right),\left|a_{j}\right|=1 \quad(j=1, \ldots, n)$, and $a_{i} \neq a_{j}(i \neq j)$, then there exists a $k$ in $A$ such that $k^{-1}$ is in $A$ and $\operatorname{Re}\left[k\left(e^{i \theta}\right) p\left(e^{i \theta}\right)\right] \geqslant 0$ a.e. on $\partial U$.

Proof. By the hypothesis on $a_{j}$, we can write $a_{j}=e^{i\left(\alpha_{j}-\pi\right)}(j=1, \ldots, n)$, where $0<\alpha_{1}<\alpha_{2}<\cdots<\alpha_{n} \leqslant 2 \pi$. Let

$$
s=\left[\frac{\sum_{j=1}^{n}\left(\alpha_{j}-\pi\right)}{4 \pi}\right] \text { and } \alpha=2 \pi\left(\frac{\sum_{j=1}^{n}\left(\alpha_{j}-\pi\right)}{4 \pi}-s\right),
$$

where $[\cdot]$ is the greatest integer function, and we have $0 \leqslant \alpha<2 \pi$. Then there exists a real valued function $\nu(\theta)$ on $[0,2 \pi]$ such that

(i) $p\left(e^{i \theta}\right) /\left|p\left(e^{i \theta}\right)\right|=e^{i \nu(\theta)}\left(0 \leqslant \theta \leqslant 2 \pi, \theta \neq \alpha_{j}, j=1, \ldots, n\right)$,

(ii) $\nu(0)=\alpha, \nu(2 \pi)-\nu(0)=2 n \pi$,

(iii) $\nu(\theta)$ is right continuous, and left continuous except for jump discontinuities of $\pi$ at $\alpha_{j}(j=1, \ldots, n)$.

Indeed, $\nu(\theta)$ has the form

$$
\nu(\theta)= \begin{cases}\alpha+j \pi+n \theta / 2 & \text { if } \alpha_{j} \leqslant \theta<\alpha_{j+1}, j=0,1, \ldots, n, \\ \alpha+2 n \pi & \text { if } \theta=2 \pi,\end{cases}
$$

where $\alpha_{0}=0$ and $\alpha_{n+1}=2 \pi$. Then there exists a continuous function $\nu_{0}$ on $[0,2 \pi]$ such that

(i) ${ }^{\prime} \nu_{0}\left(\alpha_{j}\right)=-\alpha+j \pi-(n / 2) \alpha_{j}(j=1, \ldots, n)$,

(ii) $\nu_{0}(0)=\nu_{0}(2 \pi)=-\alpha$,

(iii) ${ }^{\prime} \nu_{0}$ is a straight line in each interval $\left[\alpha_{j}, \alpha_{j+1}\right](j=0, \ldots, n)$.

Now we can find the desired function $k$ of the lemma. Let $\nu_{0}^{*}$ be the harmonic conjugate of $\nu_{0}$; then $\nu_{0}+i \nu_{0}^{*}$ belongs to $A$ because $\nu_{0}$ is in a Lipschitz class (cf. [3, p. 140]). Let $k=-i \exp \left(-\nu_{0}^{*}+i \nu_{0}\right)$; then both $k$ and $k^{-1}$ are in $A$ and

$$
\frac{k\left(e^{i \theta}\right) p\left(e^{i \theta}\right)}{\left|k\left(e^{i \theta}\right) p\left(e^{i \theta}\right)\right|}=e^{i\left(\nu(\theta)+\nu_{0}(\theta)-\pi / 2\right)}
$$

with $-\pi / 2 \leqslant \nu(\theta)+\nu_{0}(\theta)-\pi / 2 \leqslant-\pi / 2(0 \leqslant \theta \leqslant 2 \pi)$. 
ThEOREM. If $p(z)=\prod_{i=1}^{n}\left(z+a_{j}\right),\left|a_{j}\right| \geqslant 1(j=1, \ldots, n)$, and $a_{i} \neq a_{j}(i \neq j)$, then for any nonconstant function $f$ in $H^{\infty}$ with $\|f\|_{\infty} \leqslant 1, p(f) /\|p(f)\|_{1}$ is an exposed point of the unit ball of $H^{1}$.

Proof. Let $\Omega_{1}=\left\{j|1 \leqslant j \leqslant n,| a_{j} \mid=1\right\}, \Omega_{2}=\left\{j|1 \leqslant j \leqslant n,| a_{j} \mid>1\right\}$, and put $p_{i}(z)=\prod_{j \in \Omega_{i}}\left(z+a_{j}\right)$, where $p_{i}(z)=1$ if $\Omega_{i}$ is empty $(i=1,2)$. By the lemma there exists a $k$ in $A$ such that $k^{-1}$ is in $A$ and $\operatorname{Re}\left[k\left(e^{i \theta}\right) p_{1}\left(e^{i \theta}\right)\right] \geqslant 0$ on $\partial U$. So, $\operatorname{Re}\left[k\left(e^{i \theta}\right) p_{1}\left(e^{i \theta}\right)\right]>0$ on $U$ by the Poisson integral representation of $h(z) p_{1}(z)$. For any nonconstant $f$ in $H^{\infty}$ with $\|f\|_{\infty} \leqslant 1, k(f(z))$ is bounded analytic in $U$, and

$$
\operatorname{Re}\left[k(f(z)) p_{2}(f(z))^{-1} p(f(z))\right]=\operatorname{Re}\left[k(f(z)) p_{1}(f(z))\right]>0
$$

on $U$, and hence $\geqslant 0$ a.e. on $\partial U$. Then, by Proposition 5(2) of [3], we have that $p(f) /\|p(f)\|_{1}$ an exposed point of $H^{1}$.

\section{REFERENCES}

1. K. deLeeuw and W. Rudin, Exposed points and extremum problems in $H^{1}$, Pacific J. Math. 8 (1958), 467-485.

2. E. Hayashi, The solution of extremal problems in $H^{1}$, Proc. Amer. Math. Soc. 93 (1985), 690-696.

3. P. Koosis, Lectures on $H^{p}$ spaces, London Math. Soc. Lecture Notes Series, No. 40, Cambridge Univ. Press, London and New York, 1980.

4. T. Nakazi, Exposed points and extremal problems in $H^{1}$, J. Funct. Anal. 53 (1983), 224-230.

5. __ Exposed points and extremal problems in $H^{1}$. II, Tôhoku Math. J. 37 (1985), 265-269.

6. The kernels of Toeplitz operators, J. Math. Soc. Japan 38 (1986), 607-616.

7. K. Yabuta, Some uniqueness theorem for $H^{p}\left(U^{n}\right)$, Tôhoku Math. J. 24 (1972), 353-357.

Department of Mathematics, Faculty of Science (General Education), Hokkaido UniverSITY, SAPPORO 060, JAPAN 